\title{
PARACOMPACT PRODUCT SPACES DEFINED BY ULTRAFILTERS OVER THE INDEX SET
}

\author{
L. BRIAN LAWRENCE
}

(Communicated by Dennis Burke)

\begin{abstract}
Let $\omega=\{0,1, \ldots\}$, and suppose that for each $i \in \omega, C_{i}$ is a compact Hausdorff space with weight $\leq c$. A filter over $\omega$ defines a topology on $\prod_{i \in \omega} C_{i}$. We prove that the continuum hypothesis implies the existence of ultrafilters over $\omega$ for which the corresponding product space on $\prod_{i \in \omega} C_{i}$ is paracompact. Moreover, we show that every P-point in $\beta \omega-\omega$ is an ultrafilter with this property. Since box products appear as closed subspaces of ultrafilter products, our theorem extends results of Mary Ellen Rudin (1972) and Kenneth Kunen (1978).
\end{abstract}

\section{INTRODUCTION AND THEOREMS}

History. The study of normality and paracompactness in box products was initiated by Mary Ellen Rudin ([R], 1972) Kenneth Kunen ([Ku], 1978) and E. K. van Douwen ([ $\left.\left.\mathrm{vD}_{1}\right], 1975\right)$. (See also the survey papers of van Douwen [ $\mathrm{vD}_{2}$ ], and Scott Williams [W].) Box products appear as closed subspaces of ultrafilter products.

The Tychonoff topology of pointwise convergence and the box topology are examples of topologies on Cartesian products defined by filters over the index set. A filter can be used to define a topology as follows: take as a base element each Cartesian product of open sets where the set of indices on which the projection is the entire coordinate space is a set belonging to the filter. Then the filter consisting of sets with a finite complement defines the Tychonoff topology, while the improper filter consisting of the entire power set defines the box topology. C. J. Knight introduced the concept of a filter topology in the original paper on box products $([\mathrm{Kn}], 1964)$.

In this paper we show that the Rudin-Kunen method for proving paracompactness in the box product generalizes to a certain class of ultrafilter products. The decomposition theory of E. Michael ([M], 1957) underlies this method.

Received by the editors February 21, 1986 and, in revised form, September 10, 1987.

1980 Mathematics Subject Classification (1985 Revision). Primary 54D18; Secondary 54B10, 54B15, 54D40, 54G10.

Key words and phrases. Paracompactness, box product, Stone-Čech remainder, P-point, Pspace. 
Proposition 1 below, due to Kunen, is at the heart of the matter, while Proposition 2 is used in an auxiliary capacity (see [Ku] for proofs of these propositions).

Proposition 1. Suppose $X$ and $Y$ are Hausdorff regular spaces where $Y$ is a quotient space on $X$ and $f: X \rightarrow Y$ is the quotient map (so the point-set of $Y$ is a partition of $X$, and for each $p \in X, f(p)$ is the partition set to which $p$ belongs). Suppose further that $f$ is closed, $f(p)$ is a Lindelöf subspace of $X$ for each $p$, and $Y$ is paracompact. Then $X$ is paracompact.

Proposition 2. Suppose $K$ is an infinite cardinal number. A Hausdorff regular space $Y$ is paracompact if $Y$ is $K$-open (i.e. every $<K$-collection of open sets has an open intersection) and $K$-Lindelöf (i.e. every open cover has a $K$ subcover). In particular, $Y$ is paracompact if $Y$ is a $\mathbf{P}$-space and $\omega_{1}$-Lindelöf. (Recall that $y$ is a $\mathbf{P}$-point if the intersection of each countable collection of neighborhoods of $y$ is itself a neighborhood of $y$ and $Y$ is a P-space if $y$ is a P-point for each $y \in Y$, which is equivalent to $\omega_{1}$-open.)

Product spaces. Suppose the index set is $\omega=\{0,1, \ldots\}$. (We will let $\mathbf{N}$ denote $\{0,1, \ldots\}$ when we use the integers to index subsets of either $\omega$ or the Cartesian product over $\omega$. .) For each $i \in \omega$, suppose $C_{i}$ is a compact Hausdorff space where the weight (i.e. the minimal cardinality of a topological base) is $\leq c$ (the cardinality of the reals). For each filter $F$ over $\omega$, let $X(F)$ be the corresponding product space on $\prod_{i \in \omega} C_{i}$, where $\prod_{i \in \omega} U_{i}$ is a basic open set if each $U_{i}$ is open in $C_{i}$, and $\left\{i \in \omega: U_{i}=C_{i}\right\} \in F$. (For example, let each $C_{i}$ be the discrete two point space $\{0,1\}$, and let $F$ be any free ultrafilter. Then in contrast to the discrete box product $\square_{i \in \omega} C_{i}$, every point of $X(F)$ is a limit point.)

Proposition 3. Suppose $F$ is a free ultrafilter over $\omega$. Then the box product $\square \square_{i \in \omega} C_{i}$ can be embedded as a closed subspace in a product space over $F$.

Proof. Let $u \in F$ where $\omega-u$ is infinite. Let $l: \omega-u \rightarrow \omega$ be a $1-1$ correspondence, and define $C^{\prime}$ so that $C_{i}^{\prime}=C_{l_{i}}$ for each $i \in \omega-u$. Let $X^{\prime}$ be the product space where $\prod_{i \in \omega} C_{i}^{\prime}$ is the point-set and the topology is defined by $F$. Let $p \in X^{\prime}$. Then $\left\{q \in X^{\prime}:(\forall i \in u)\left(q_{i}=p_{i}\right)\right\}$ is a closed subspace of $X^{\prime}$ that is homeomorphic to $\square_{i \in \omega} C_{i}$.

An alternative definition of $C^{\prime}$ (due to the referee) is to let $C_{i}^{\prime}$, for each $i \in \omega$, be the one point compactification of the topological sum $\bigcup_{i \in \omega} C_{i}$.

Quotient spaces. For each $p \in \prod_{i \in \omega} C_{i}$, define the derived sequence $E=E(p)$ on $\mathbf{N}$ by $E_{m}=\left\{q \in \prod_{i \in \omega} C_{i}:(\forall i>m)\left(q_{i}=p_{i}\right)\right\}$, and define the derived set to be $e(p)=\bigcup_{m \in \mathbf{N}} E_{m}$. Note that the image of $e$ is a partition of the product. For each filter $F$ over $\omega$, let $Y(F)$ be the quotient space on $X(F)$ induced by $e$.

Then regardless of the choice of $F$, we have the following results. (1) For each $p, E(p)$ is a sequence of compact sets in $X(F)$, so $e(p)$ is a Lindelöf 
subspace. (2) As a quotient map, $e$ is open; so the weight of $Y(F)$ does not exceed that of $X(F)$ which is $\leq c$. Thus by Proposition 2, the continuum hypothesis implies that $Y(F)$ is paracompact if it is a $\mathbf{P}$-space.

P-point ultrafilters. We view the free ultrafilters over $\omega$ as the point-set of $\beta \omega-\omega$ (the remainder of the Stone-Čech compactification of the integers). Recall that for $F \in \beta \omega-\omega, F$ is a P-point iff $(\forall \theta: \mathbf{N} \rightarrow \mathbf{F}) \quad(\exists u \in F)(\forall n \in$ $\mathbf{N})\left(u-\theta_{n}\right.$ is finite). We use this characterization to prove Theorems 1 and 2 below.

Theorem 1. Suppose $F \in \beta \omega-\omega$. Then the quotient space $Y(F)$ is a $\mathbf{P}$-space iff $F$ is a $\mathbf{P}$-point.

Theorem 2. Suppose $F \in \beta \omega-\omega$. Then the quotient map $e: X(F) \rightarrow Y(F)$ is closed iff $F$ is a P-point.

Main theorem. The continuum hypothesis implies that for every $\mathbf{P}$-point $F \in$ $\beta \omega-\omega, X(F)$ is paracompact. (In light of the first two propositions, the main theorem is a corollary of Theorems 1 and 2.)

\section{Notation}

Basic open sets. Suppose $F \in \beta \omega-\omega$. Two parameters govern the structure of a basic open set in $X(F)$. The first is a choice of a basic open set in the box topology called an open box. Suppose $A_{i}$ is a proper open subset of $C_{i}$ for each $i \in \omega$. The second is a choice of a filter set. Suppose $u \in F$. Define $U=U(A, u)$ by $U_{i}=C_{i}$ if $i \in u$, and $U_{i}=A_{i}$ otherwise. (We use $U$ to denote both the set-valued sequence $\left(U_{i}\right)$ and the Cartesian product $\prod_{i \in \omega} U_{i}$.) So we can view a basic open set in $X(F)$ as a Cartesian product built up from a core open box.

Diagonal sequences. Suppose $F \in \beta \omega-\omega$ and $p \in X(F)$. In analogy with the definition of the derived sequence of $p$, we can define the derived sequence $\left(V_{m}\right)$ of an open set $U$ containing $p$. (See the following definitions.)

Now suppose we are given a sequence of basic open sets $\left(U_{n}\right)$ in $X(F)$ each containing $p$. Build an infinite matrix of open sets in $X(F)$ by placing the derived sequence $\left(V_{n, m}\right)$ of $U_{n}$ in the $n$th row. To prove that the quotient space is a $\mathbf{P}$-space, we need a diagonal sequence $\left(V_{n, j_{n}}\right)$ where $j: \mathbf{N} \rightarrow \mathbf{N}$ is strictly increasing and the intersection is open; and to prove that $e$ is closed (by way of the upper semicontinuous decomposition theorem), we need a subsequence $\left(V_{j_{n}, j_{n}}\right)$ of the main diagonal where the intersection is open. In either case, the point of departure from the box topology is that the intersection of a diagonal sequence is open iff the intersection of the underlying sequence of filter sets is itself a filter set. We show that for an ultrafilter $F$, the relevant intersection property is precisely that which characterizes a P-point in $\beta \omega-\omega$.

Index set. For each $n \in \mathbf{N}$, let $\sigma_{n}$ be the initial segment $\{0, \ldots, n\}$, and let $\tau_{n}$ be the tail end $\{n+1, \ldots\}$. 
Hypothesis. For the definitions given below, suppose $F \in \beta \omega-\omega, \theta: \mathbf{N} \rightarrow F$, and $U(A, u)$ is a basic open set in $X(F)$.

Derived sequence and set. Define the derived sequence of $U$ to be $V=V(A, u)$ where for each $m, V_{m, i}=C_{i}$ if $i \in \sigma_{m} \cup u$, and $V_{m, i}=A_{i}$ otherwise. Define the derived set $W=W(A, u)$ by $W=\bigcup_{m \in \mathbf{N}} V_{m}$. Then $W=\bigcup\{e(p): p \in U\}$.

Excised derived sequence and set. Define the excised set $U^{\circ}=U^{\circ}(A, u)$ by $U_{i}^{\circ}=C_{i}-A_{i}$ if $i \in u$, and $U_{i}^{\circ}=A_{i}$ otherwise. Define the excised derived sequence $V^{\circ}=V^{\circ}(A, u)$ by $V_{m, i}^{\circ}=C_{i}-A_{i}$ if $i \in u$, and $V_{m, i}^{\circ}=V_{m, i}$ otherwise. Let $W^{\circ}=W^{\circ}(A, u)$ be the union of the terms of $V^{\circ}$. $W^{\circ}$ is the excised derived set. Note that $U^{\circ} \subseteq U, U^{\circ} \subseteq V_{m}^{\circ} \subseteq V_{m}$ for each $m$, and $W^{\circ} \subseteq W$.

Basic diagonal sequence. A sequence of basic open sets in $X(F)$ is in basic diagonal form if it can be represented as $D(A, \theta)$ for some $A$ and $\theta$. Define $D=D(A, \theta)$ by $D_{n, i}=C_{i}$ if $i \in \sigma_{n} \cup \theta_{n}$, and let $D_{n, i}=A_{i}$ otherwise.

\section{PROOF OF THEOREM 1}

Suppose $Y(F)$ is a $\mathbf{P}$-space, $\theta: \mathbf{N} \rightarrow F$, and $A$ is an open box. For each $n \in \mathbf{N}$, let $U_{n}=U\left(A, \theta_{n}\right)$, let $\left(V_{n, m}\right)$ be the associated derived sequence, and let $W_{n}$ be the derived set. By hypothesis, $\bigcap_{n \in \mathbf{N}} W_{n}$ is open. Let $B=U(A, b)$ be a basic open set contained in the intersection, and let $B^{0}=U^{0}(A, b)$ be the excised set. For each $n$, let $s_{n}=b-\theta_{n}$.

Claim. Each $s_{n}$ is a finite set. Fix $n$. Since $B^{0} \subseteq W_{n}=\bigcup_{m \in \mathbf{N}} V_{n, m}$, there exists $j_{n}$ with $B^{0} \subseteq V_{n, j_{n}}$ (if $B^{0}$ intersects $V_{n, j_{n}}$, then $B^{\circ} \subseteq V_{n, j_{n}}$ ). For $i \in \tau_{j_{n}}, V_{n, j_{n}, i}=U_{n, i}$, and since $V_{n, j_{n}}$ disagrees with $U_{n}$ on each index in $s_{n}$ (by the choice of $s$ and the preceding inclusion), we have $s_{n} \subseteq \sigma_{j_{n}}$ which is a finite set.

For the converse, suppose $F$ is a $\mathbf{P}$-point. Suppose $U$ is a sequence of basic open sets and let $\theta$ be the sequence in $F$ that underlies $U$. Let $t \in F$ where $s_{n}=t-\theta_{n}$ is finite for each $n$. Let $p \in \bigcap_{n \in \mathbf{N}} W_{n}$ where $W_{n}$ is the derived set of $U_{n}$. Then we can choose a strictly increasing sequence $j: \mathbf{N} \rightarrow \mathbf{N}$ such that for each $l \in \mathbf{N}$ and each $i \in \tau_{j_{l}}, p_{i} \in \bigcap_{n \leq l} U_{n, i}$. Let $B_{i}=\bigcap_{n \leq l} U_{n, i}$ if $i \in \tau_{j_{l}}-\tau_{j_{l+1}}$ and $i \in \omega-t$, and otherwise, let $B_{i}=C_{i}$.

Claim. $B \subseteq \bigcap_{n \in \mathbf{N}} W_{n}$. Fix $n$. Choose $m$ so that $m \geq j_{n}$ and $s_{n} \subseteq \sigma_{m}$. Since $s_{n} \subseteq \sigma_{m}$, if $i \in \tau_{m}$, then $U_{n, i}=C_{i}$ or $i \in \omega-t$. In either case, since $m \geq j_{n}$, $B_{i} \subseteq U_{n, i}=V_{n, m, i}$. For $i \in \sigma_{m}, B_{i} \subseteq C_{i}=V_{n, m, i}$.

\section{Proof of Theorem 2}

Lemma 1. The quotient map $e: X(F) \rightarrow Y(F)$ is closed iff every basic diagonal sequence has a subsequence with an open intersection.

Claim 1. If $p \in X(F)$ and $S$ is a basic open cover of $e(p)$ (as a subspace of $X(F))$, then there is a basic diagonal sequence $D=D(A, \theta)$ such that $p \in A$, and $\bigcup_{n \in \mathbf{N}} D_{n} \subseteq \cup S$. 
Proof. Let $E$ be the derived sequence of $p$. By the compactness of the coordinate spaces, $E$ is a sequence of compact sets, so we can choose a sequence $T$ of finite subcollections of $S$ such that each $T_{n}$ is a cover of $E_{n}$. We assume that if $U \in T_{n}$, then $U$ intersects $E_{n}$; equivalently, $p_{i} \in U_{i}$ for $i \in \tau_{n}$. For each $i \in \omega$, let $A_{i}$ be a proper open subset of $C_{i}$ such that $p_{i} \in A_{i}$ and $A_{i} \subseteq \bigcap\left\{U_{i}: U \in T_{n}\right.$ for some $\left.n<i\right\}$. Let $\theta: \mathbf{N} \rightarrow F$ defined by $\theta_{n}=\left\{i: U_{i}=C_{i}\right.$ for each $\left.U \in T_{n}\right\}$. Let $D=D(A, \theta)$. Then for each $n, D_{n} \subseteq \cup T_{n}$ by the choice of $A$ and $\theta$.

Claim 2. Suppose $j: \mathbf{N} \rightarrow \mathbf{N}$ is strictly increasing. If $q \in \bigcap_{n \in \mathbf{N}} D_{j_{n}}$, then $e(q) \subseteq \bigcup_{n \in \mathbf{N}} D_{j_{n}}$; i.e. the derived set of $\bigcap_{n \in \mathbf{N}} D_{j_{n}}$ is a subset of $\bigcup_{n \in \mathbf{N}} D_{j_{n}}$.

Proof. Let $r \in e(q)$. Let $l \in \mathbf{N}$ with $r_{i}=q_{i}$ for each $i \in \tau_{l}$. We have $q \in \bigcap_{n \in \mathrm{N}} D_{j_{n}}$, so $q \in D_{j_{l}}$, which implies $r \in D_{j_{l}}$, since $\sigma_{l} \subseteq \sigma_{j_{l}}$ and $D_{j_{l}, i}=C_{i}$ for each $i \in \sigma_{j_{l}}$.

Remark. In contrast to the analogue for the box topology, the terms of $D \circ j$ are incomparable with respect to set inclusion and the derived set of the intersection is a proper subset of the union which is not itself a derived set.

Claim 3. Suppose $D=D(A, \theta)$ is a basic diagonal sequence where each term is a proper subset of $\prod_{i \in \omega} C_{i}$. Suppose $W=W(A, u)$ is a derived set with $W \subseteq \bigcup_{n \in \mathrm{N}} D_{n}$. Then the terms of $D$ that intersect the excised derived set $W^{\circ}$ are infinite in number and their intersection is open.

Proof.

Subclaim 1. For each $m \in \mathbf{N}, W^{\circ}$ intersects the complement of $\bigcup_{n \leq m} D_{n}$. Choose $k$ so that for each $n \leq m$, there exists $l_{n} \in \sigma_{k}$ with $D_{n, l_{n}}=A_{l_{n}}$. Let $V^{\circ}=V^{\circ}(A, u)$. Let $p \in V_{k}^{\circ}$ where $p_{i} \in C_{i}-A_{i}$ for each $i \in \sigma_{k}$. Then $p \in W^{\circ}-\bigcup_{n \leq m} D_{n}$.

Subclaim 2. Let $D \circ j$ be the subsequence of $D$ consisting of those terms that intersect $W^{\circ}$; then $D_{j_{n}, i}=C_{i}$ for each $i \in u$. This implies $U(A, u) \subseteq$ $\bigcap_{n \in \mathbf{N}} D_{j_{n}}$.

Proof of Lemma 1. By the U.S.C. Decomposition Theorem and Claim 1, a closed quotient map is equivalent to the condition that for every basic diagonal sequence $D=D(A, \theta)$, there exists $u \in F$ such that $W(A, u)$ is a subset of $\bigcup_{n \in \mathrm{N}} D_{n}$. In light of this relationship, sufficiency follows from Claim 2 and necessity from Claim 3.

Lemma 2. Every basic diagonal sequence has a subsequence with an open intersection iff $F$ is a P-point.

Proof. First note that for any basic diagonal sequence $D=D(A, \theta)$, the intersection of a subsequence, $\bigcap_{n \in \mathbf{N}} D_{j_{n}}$, is a Cartesian product where the $i$ th factor is either $A_{i}$ or $C_{i}$. The $i$ th factor is $C_{i}$ iff $i \in \bigcap_{n \in \mathbf{N}}\left(\sigma_{j_{n}} \cup \theta_{j_{n}}\right)$.

Suppose diagonal sequences behave appropriately, and let $\theta: \mathbf{N} \rightarrow F$. Suppose $u \in F$ so that $u$ is almost contained (i.e. the difference is finite) in each 
of $\theta_{0}, \theta_{0} \cap \theta_{1}, \theta_{0} \cap \theta_{1} \cap \theta_{2}, \ldots$. Then $u$ is almost contained in each term of the original sequence. So we can assume that $\theta$ is monotone nonincreasing.

Choose an open box $A$ arbitrarily and let $D=D(A, \theta)$. Let $j: \mathbf{N} \rightarrow \mathbf{N}$ where $\bigcap_{n \in \mathbf{N}} D_{j_{n}}$ is open. Let $u=\bigcap_{n \in \mathbf{N}}\left(\sigma_{j_{n}} \cup \theta_{j_{n}}\right)$. Then $u \in F$. Since $\sigma_{j_{n}}$ is finite for each $n, u$ is almost contained in each term of the subsequence. By the monotone property, this result also holds for the original sequence.

For the converse, suppose $F$ is a P-point and let $D=D(A, \theta)$ be a basic diagonal sequence. Let $t \in F$ where $t$ is almost contained in each term of $\theta$. Choose a strictly increasing sequence $j: \mathbf{N} \rightarrow \mathbf{N}$ where for each $n \in \mathbf{N}$, $t \cap \tau_{j_{n+1}} \subseteq \theta_{j_{n}}$. Note that for each $n \in \mathbf{N}$ and each $i \in \omega$, if $i \in t \cap \sigma_{j_{n}}$ or $i \in t \cap \tau_{j_{n+1}}$, then $D_{j_{n}, i}=C_{i}$.

Partition $\omega$ into intervals by defining interval $n$ to be $\sigma_{j_{n}}-\sigma_{j_{n-1}}$. Let $t$-odd ( $t$-even) be the elements of $t$ that belong to an odd-numbered (even-numbered) interval. Either $t$-odd or $t$-even belongs to $F$. If $t$-odd $\in F(t$-even $\in F)$, then let $u$ be the set of indices on which the projection of

$$
\bigcap_{n \in \mathbf{N}} D_{j_{2 n+1}}\left(\bigcap_{n \in \mathbf{N}} D_{j_{2 n}}\right)
$$

is the entire coordinate space; by the observation of the preceding paragraph, it follows that $t$-odd $\subseteq u \quad(t$-even $\subseteq u)$, so the intersection is open.

\section{ACKNOWLEDGMENTS}

A key step in the proof of Theorem 2 is due to Kenneth Kunen (specifically, the proof of sufficiency for Lemma 2). In the original version of this paper, the main theorem held only for selective P-points. The improvement is a consequence of Kunen's observation.

This paper is partially based on Part II-Chapters 3 and 4 of the author's Ph.D. thesis, State University of New York at Binghamton. The author thanks his thesis advisor Professor Prabir Roy.

The author also thanks the referee for many helpful comments and points of reference.

\section{REFERENCES}

[ $\mathrm{vD}_{1}$ ] E. K. van Douwen, The box product of countably many metrizable spaces need not be normal, Fund. Math. 88 (1975), 127-132.

$\left[\mathrm{vD}_{2}\right] \ldots$, Covering and separation in box products, Surveys in General Topology, Academic Press, New York, 1980, pp. 55-103.

[Kn] C. J. Knight, Box topologies, Quart. J. Math. Oxford Ser. (2) 15 (1964), 41-54.

[Ku] K. Kunen, On paracompactness of box products of compact spaces, Trans. Amer. Math. Soc. 240 (1978), 307-316.

[M] E. Michael, Another note on paracompact spaces, Proc. Amer. Math. Soc. 8 (1957), 822-828. 
[R] M. E. Rudin, The box product of countably many compact metric spaces, General Topol. Appl. 2 (1972), 293-298.

[W] S. Williams, Box products, Handbook of Set-Theoretic Topology, K. Kunen, J. Vaughan, eds., Elsevier, New York, 1984 pp. 169-200.

Howard University, Washington, District of Columbia 20059

Current address: George Mason University, Fairfax, Virginia 22030 